\title{
The ANTARES telescope neutrino alert system
}

\author{
M. Ageron ${ }^{\text {a }}$, J.A. Aguilar ${ }^{\text {b }}$, I. Al Samarai ${ }^{\text {a }}$, A. Albert ${ }^{\text {c }}$, M. André ${ }^{\text {d }}$, M. Anghinolfi ${ }^{\text {, }}$, G. Anton ${ }^{f}$, S. Anvar ${ }^{\mathrm{g}}$,
} M. Ardid ${ }^{\text {h }}$, A.C. Assis Jesus ${ }^{\mathrm{i}}$, T. Astraatmadja ${ }^{\mathrm{i}, 1}$, J.-J. Aubert ${ }^{\mathrm{a}}$, B. Baret ${ }^{\mathrm{j}}$, S. Basa ${ }^{\mathrm{k}}$, V. Bertin $^{\text {a }}$, S. Biagi ${ }^{1, \mathrm{~m}}$, A. Bigi ${ }^{n}$, C. Bigongiari ${ }^{b}$, C. Bogazzi ${ }^{i}$, M. Bou-Cabo ${ }^{h}$, B. Bouhou ${ }^{j}$, M.C. Bouwhuis ${ }^{i}$, J. Brunner $^{a, 2}$, J. Busto $^{a}$, F. Camarena ${ }^{\mathrm{h}}$, A. Capone ${ }^{\mathrm{o}, \mathrm{p}}$, C. Cârloganu ${ }^{\mathrm{q}}$, G. Carminati ${ }^{\mathrm{l}, \mathrm{m}, 3}$, J. Carr $^{\mathrm{a}}$, S. Cecchini ${ }^{1}$, Z. Charif $^{\mathrm{a}}$, Ph. Charvis ${ }^{\mathrm{r}, \mathrm{s}}$, T. Chiarusi ${ }^{1}$, M. Circella ${ }^{t}$, R. Coniglione ${ }^{v}$, H. Costantini ${ }^{\text {e,a }}$, P. Coyle ${ }^{a}$, C. Curtil $^{a}$, M.P. Decowski ${ }^{i}$, I. Dekeyser ${ }^{\mathrm{u}}$,

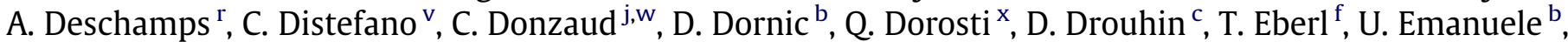
A. Enzenhöfer ${ }^{f}$, J-P. Ernenwein ${ }^{a}$, S. Escoffier ${ }^{a}$, P. Fermani ${ }^{o, p}$, M. Ferri ${ }^{h}$, V. Flaminio ${ }^{n, y}$, F. Folger ${ }^{f}$, U. Fritsch ${ }^{f}$, J-L. Fuda ${ }^{\mathrm{u}}$, S. Galatà a, P. Gay ${ }^{\mathrm{q}}$, G. Giacomelli ${ }^{1, \mathrm{~m}}$, V. Giordano ${ }^{\mathrm{v}}$, J.P. Gómez-González ${ }^{\mathrm{b}}$, K. Graf ${ }^{\mathrm{f}}$, G. Guillard ${ }^{\mathrm{q}}$, G. Halladjian ${ }^{\text {a }}$, G. Hallewell ${ }^{\text {a }}$, H. van Haren ${ }^{\text {z }}$, J. Hartman ${ }^{i}$, A.J. Heijboer ${ }^{\text {i }}$, Y. Hello ${ }^{\text {r,s }}$, J.J. Hernández-Rey ${ }^{\text {b }}$, B. Herold ${ }^{f}$, J. Hößl ${ }^{f}$, C.C. Hsu ${ }^{i}$, M. de Jong ${ }^{i, 1}$, M. Kadler ${ }^{\text {aa }}$, O. Kalekin ${ }^{f}$, A. Kappes ${ }^{f}$, U. Katz ${ }^{f}$, O. Kavatsyuk ${ }^{\mathrm{x}}$, P. Kooijman ${ }^{\text {i,ab,ac }}$, C. Kopper ${ }^{\text {f }}$, A. Kouchner ${ }^{j}$, I. Kreykenbohm ${ }^{\text {aa }}$, V. Kulikovskiy ${ }^{\text {ad,e, }}$, R. Lahmann ${ }^{f}$, P. Lamare ${ }^{g}$,

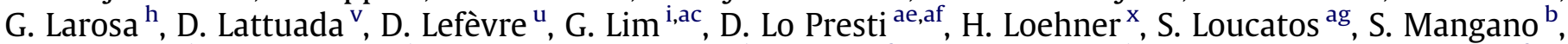
M. Marcelin ${ }^{\text {k }}$, A. Margiotta ${ }^{\text {l,m }}$, J.A. Martínez-Mora ${ }^{\text {h }}$, A. Meli ${ }^{\text {f, T. Montaruli }}{ }^{\text {t,ah }}$, L. Moscoso ${ }^{\text {j,ag, }, 4}$, H. Motz $^{\mathrm{f}}$, M. Neff ${ }^{f}$, E. Nezri ${ }^{k}$, D. Palioselitis ${ }^{i}$, G.E. Păvălaş ${ }^{a i}$, K. Payet ${ }^{\text {ag }}$, P. Payre ${ }^{\mathrm{a}, 4}$, J. Petrovic ${ }^{i}$, P. Piattelli ${ }^{\mathrm{v}}$, N. PicotClemente $^{a}$, V. Popa ${ }^{\text {ai }}$, T. Pradier ${ }^{\text {aj }}$, E. Presani ${ }^{i}$, C. Racca $^{c}$, C. Reed ${ }^{i}$, C. Richardt ${ }^{f}$, R. Richter $^{f}$, C. Rivière ${ }^{a}$, A. Robert ${ }^{t}$, K. Roensch $^{\mathrm{f}}$, A. Rostovtsev ${ }^{\mathrm{ak}}$, J. Ruiz-Rivas ${ }^{\mathrm{b}}$, M. Rujoiu ${ }^{\mathrm{ai}}$, G.V. Russo ${ }^{\text {ae,af }}$, F. Salesa ${ }^{\mathrm{b}}$, P. Sapienza ${ }^{\mathrm{v}}$, F. Schöck ${ }^{f}$, J-P. Schuller ${ }^{\text {ag }}$, F. Schüssler ${ }^{\text {ag }}$, R. Shanidze ${ }^{f}$, F. Simeone ${ }^{o, p}$, A. Spies ${ }^{f}$, M. Spurio ${ }^{1, m}$, J.J.M. Steijger ${ }^{i}$, Th. Stolarczyk ${ }^{\text {ag }}$, A. Sánchez-Losa ${ }^{b}$, M. Taiuti ${ }^{e},{ }^{a l}$, C. Tamburini ${ }^{\mathrm{u}}$, S. Toscano $^{\mathrm{b}}$, , B. Vallage $^{\text {ag }}$, V. Van Elewyck $^{\mathrm{j}}$,

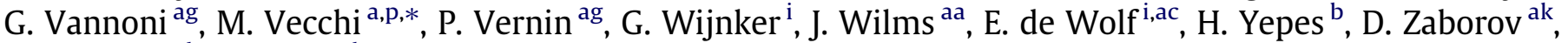
J.D. Zornoza ${ }^{\text {b }}$, J. Zúñiga ${ }^{b}$

${ }^{a}$ CPPM, Aix-Marseille Université, CNRS/IN2P3, Marseille, France

${ }^{\mathrm{b}}$ IFIC - Instituto de Física Corpuscular, Edificios Investigación de Paterna, CSIC, Universitat de València, Apdo. de Correos 22085,46071 Valencia, Spain ${ }^{\mathrm{C}}$ GRPHE - Institut universitaire de technologie de Colmar, 34 rue du Grillenbreit BP50568, 68008 Colmar, France

${ }^{\mathrm{d}}$ Technical University of Catalonia, Laboratory of Applied Bioacoustics, Rambla Exposició, 08800 Vilanova i la Geltrú, Barcelona, Spain

e INFN - Sezione di Genova, Via Dodecaneso 33, 16146 Genova, Italy

${ }^{\mathrm{f}}$ Friedrich-Alexander-Universität Erlangen-Nürnberg, Erlangen Centre for Astroparticle Physics, Erwin-Rommel-Str. 1, 91058 Erlangen, Germany

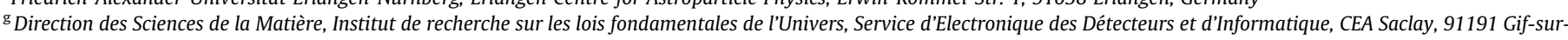
Yvette Cedex, France

${ }^{\mathrm{h}}$ Institut d'Investigació per a la Gestió Integrada de les Zones Costaneres (IGIC), Universitat Politècnica de València. C/ Paranimf 1, 46730 Gandia, Spain

${ }^{\mathrm{i}}$ Nikhef, Science Park, Amsterdam, The Netherlands

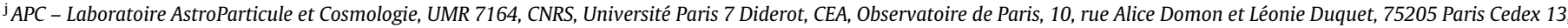
France

${ }^{\mathrm{k}}$ LAM - Laboratoire d'Astrophysique de Marseille, Pôle de l'Étoile Site de Château-Gombert, rue Frédéric Joliot-Curie 38, 13388 Marseille Cedex 13, France

${ }^{1}$ INFN - Sezione di Bologna, Viale Berti Pichat 6/2, 40127 Bologna, Italy

${ }^{\mathrm{m}}$ Dipartimento di Fisica dell'Università, Viale Berti Pichat 6/2, 40127 Bologna, Italy

${ }^{n}$ INFN - Sezione di Pisa, Largo B. Pontecorvo 3, 56127 Pisa, Italy

o INFN - Sezione di Roma, P.le Aldo Moro 2, 00185 Roma, Italy

${ }^{\mathrm{P}}$ Dipartimento di Fisica dell'Università La Sapienza, P.le Aldo Moro 2, 00185 Roma, Italy

${ }^{\mathrm{q}}$ Clermont Université, Université Blaise Pascal, CNRS/IN2P3, Laboratoire de Physique Corpusculaire, BP 10448, 63000 Clermont-Ferrand, France

${ }^{\mathrm{r}}$ Géoazur, Université de Nice Sophia-Antipolis, CNRS/INSU, IRD, Observatoire de la Côte d'Azur, France

${ }^{s}$ Université Pierre et Marie Curie, BP 48, 06235 Villefranche-sur-mer, France

t INFN - Sezione di Bari, Via E. Orabona 4, 70126 Bari, Italy

" COM - Centre d'Océanologie de Marseille, CNRS/INSU et Université de la Méditerranée, 163 Avenue de Luminy, Case 901, 13288 Marseille Cedex 9, France

${ }^{v}$ INFN - Laboratori Nazionali del Sud (LNS), Via S. Sofia 62, 95123 Catania, Italy

${ }^{\mathrm{w}}$ Univ Paris-Sud, 91405 Orsay Cedex, France

${ }^{\mathrm{x}}$ Kernfysisch Versneller Instituut (KVI), University of Groningen, Zernikelaan 25, 9747 AA Groningen, The Netherlands

${ }^{y}$ Dipartimento di Fisica dell'Università, Largo B. Pontecorvo 3, 56127 Pisa, Italy

${ }^{\mathrm{z}}$ Royal Netherlands Institute for Sea Research (NIOZ), Landsdiep 4,1797 SZ 't Horntje (Texel), The Netherlands

${ }^{\text {aa }}$ Dr. Remeis-Sternwarte and ECAP, Universität Erlangen-Nürnberg, Sternwartstr. 7, 96049 Bamberg, Germany

${ }^{\mathrm{ab}}$ Universiteit Utrecht, Faculteit Betawetenschappen, Princetonplein 5, 3584 CC Utrecht, The Netherlands 


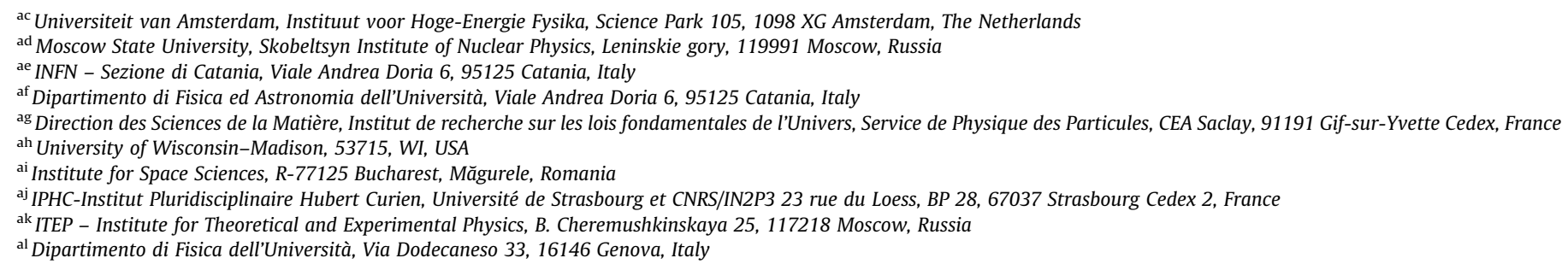

\section{A R T I C L E I N F O}

Article history:

Received 11 March 2011

Received in revised form 3 November 2011

Accepted 29 November 2011

Available online 20 December 2011

\section{Keywords:}

ANTARES

Neutrino astronomy

Transient sources

Optical follow-up

\begin{abstract}
A B S T R A C T
The ANTARES telescope has the capability to detect neutrinos produced in astrophysical transient sources. Potential sources include gamma-ray bursts, core collapse supernovae, and flaring active galactic nuclei. To enhance the sensitivity of ANTARES to such sources, a new detection method based on coincident observations of neutrinos and optical signals has been developed. A fast online muon track reconstruction is used to trigger a network of small automatic optical telescopes. Such alerts are generated for special events, such as two or more neutrinos, coincident in time and direction, or single neutrinos of very high energy.
\end{abstract}

(ㄷ) 2011 Elsevier B.V. All rights reserved.

\section{Introduction}

The detection of high energy cosmic neutrinos from a source would be direct evidence of the presence of hadronic acceleration within the source and provide important information on the origin of the high energy cosmic rays. Powerful sources of transient nature, such as gamma ray bursts or core collapse supernovae, offer one of the most promising perspectives for the detection of cosmic neutrinos as, due to their short duration, they are essentially background free. For example, several authors predict the emission of neutrinos in correlation with multi-wavelength signals, e.g. the Fireball model of GRBs [1]. As neutrino telescopes observe a full hemisphere of the sky (even the whole sky if downgoing events are considered) at all times, they are particularly well suited for the detection of transient phenomena.

In this paper, the implementation of a strategy for the detection of transient sources is presented. This method, earlier proposed in [2], is based on the optical follow-up of selected neutrino events very shortly after their detection (Section 3 ) by the ANTARES neutrino telescope [3]. The alert system, known as "TAToO" (Telescopes and ANTARES Target of Opportunity) [4], uses an online track reconstruction with a pointing accuracy of about 0.5 degrees. This reconstruction algorithm is described in Section 4. Its characteristics allow the triggering of small robotic optical telescopes such as those of TAROT [5] and ROTSE-III [6], which have slewing and settling time below $10 \mathrm{~s}$. TAROT consists of two $25 \mathrm{~cm}$ telescopes with a large field of view of $1.98^{\circ} \times 1.98^{\circ}$ and magnitude limit of 18-19, located at Calern, France, and La Silla, Chile. ROTSE-III is an array of four $45 \mathrm{~cm}$ telescopes with field of view of $1.86^{\circ} \times 1.86^{\circ}$ and magnitude limit of 19 , located at Sidings Springs Observatory, Australia, McDonald Observatory, Texas, Mt. Gamsberg, Namibia, and Bakirlitepe, Turkey. In order to improve the precision of these alerts, an additional reconstruction algo-

\footnotetext{
* Corresponding author at: CPPM, Aix-Marseille Université, CNRS/IN2P3, Marseille, France.

E-mail address: vecchi@cppm.in2p3.fr (M. Vecchi)

1 Also at University of Leiden, the Netherlands.

2 On leave at DESY, Platanenallee 6, D-15738 Zeuthen, Germany.

${ }^{3}$ Now at University of California - Irvine, 92697, CA, USA.

4 Deceased.
}

rithm, described in Section 5, which takes into account the detailed detector geometry is used offline.

\section{The ANTARES detector and data acquisition}

The ANTARES neutrino telescope is located in the Mediterranean Sea, $40 \mathrm{~km}$ from the coast of Toulon, France, at a depth of $2475 \mathrm{~m}$. The detector is an array of photomultiplier tubes (PMTs) arranged on 12 slender detection lines, anchored to the sea bed and kept taut by a buoy at the top. Each line comprises up to 25 storeys of triplets of optical modules (OMs), each housing a single $10^{\prime \prime}$ PMT. Since lines are subject to the sea current and can change shape and orientation, a positioning system comprising hydrophones and compass-tiltmeters is used to monitor the detector geometry. Data taking started in 2006 with the operation of the first line of the detector. The construction of the 12 line detector was completed in May 2008. The main goal of the experiment is to search for neutrinos of astrophysical origin by detecting high energy muons $(\geqslant 100 \mathrm{GeV}$ ) induced by their neutrino charged current interaction in the vicinity of the detector. Due to the large background from downgoing cosmic ray induced muons, the detector is optimized for the detection of upgoing neutrino induced muon tracks.

\subsection{Data acquisition}

The task of the ANTARES data acquisition system (DAQ) [7] is to collect the data from all the individual PMTs of the detector and pass them to the filtering algorithms which search for a collection of signals compatible with a muon track crossing the detector.

From the DAQ point of view, each storey is an independent acquisition unit including a processor, buffering RAM and an Ethernet link to the shore station. Individual PMT pulses above a threshold of typically 0.3 photoelectron (referred to as 'hits') are digitized offshore in the form of 'hit time' and 'charge', and sent to a computer farm onshore for further processing. Due to bioluminescence activity and ${ }^{40} \mathrm{~K}$ decays in the sea water, each PMT has an average counting rate of the order of $100 \mathrm{kHz}$, requiring large bandwidth for data transmission to shore. The data are transmitted through gigabit Ethernet links on single optical fibers to the computing farm where the filtering algorithms are executed, reducing the 
event rate to a few tens of hertz. The overall system is supervised by a state machine which handles the various commands needed to configure, start and stop the acquisition on both the offshore and onshore processors.

Data are time structured in the form of time slices of $104.85 \mathrm{~ms}$, allowing the data of the full detector for the duration of one time slice to be sent to a single computing node. The synchronization of the 300 offshore processors is performed by a $20 \mathrm{MHz}$ clock distribution system broadcasted to all storeys. In particular, the start of any data taking period is stamped using an external GPS signal giving the absolute timing at the location of the detector, allowing an absolute time accuracy better than $1 \mu$ s [8].

\subsection{Data filtering}

The main goal of the filtering algorithm is to select hits compatible with the propagation of Cherenkov light emitted by a muon crossing the detector, among the background from bioluminescence and ${ }^{40} \mathrm{~K}$ decays characterized by uncorrelated hits on single OMs. The filtering is based on local 'clusters', defined either as coincidence hits on OMs of the same storey within a narrow time window or as a single hit with a large amplitude. There are two main filtering algorithms running simultaneously, both searching for a combination of local clusters within a typical $2.2 \mu$ s time window. The first algorithm requires five local causally connected clusters anywhere in the detector, while the second requires at least two local clusters in adjacent or next-to-adjacent storeys. All hits within a few microseconds around these clusters define an "event" and are kept for further online and offline reconstructions.

\subsection{The TAToO trigger}

Once a single data filtering computer has processed its time slice, the resulting events are sent to a data distribution service to which the "Data Writer" storing task, monitoring tasks and the online event reconstruction [9] are connected. The "alert" application described in Section 4 analyses the data stream of reconstructed events, selects candidates fulfilling various criteria, and then generates the TAToO alerts.

An important performance parameter for the alert system is the time between the crossing of the detector by a high energy muon and the time at which an alert is sent. This time is the sum of the data dispatching time from the offshore photomultipliers to the onshore computing farm (1.5 s) and of the data processing time of an entire time slice by the filtering algorithm $(\leqslant 5 \mathrm{~s})$. For the time-being, the data transmission to the processors suffers from an additional latency of up to one minute, which will be removed after an upgrade of the acquisition to improve the performance of the alert sending capability. The time needed to reconstruct the event direction and verify the alert criteria typically amounts to a few milliseconds. Therefore, the total delay between an interesting particle crossing the detector and a TAToO alert is currently about one minute.

\section{The TAToO run control}

The TAToO Run Control (RC) is a stand-alone Qt control application $^{5}$ which channels the triggers generated by the alert application to the optical telescope network.

The connections to this network are checked periodically and automatic reconnection is performed resulting in a fully autonomous and stable system. A veto prevents an alert to be sent if the ANTARES event counting rate exceeds a given threshold. In addition, if the alert criteria are fulfilled soon after a previous alert has

\footnotetext{
${ }^{5}$ Software framework originally from Trolltech, now Nokia.
}

already been issued, the new alert is stored in a FIFO and sent only after a certain period of time. This time lag, currently set at one hour, is used to avoid alert pileup in the optical telescope network. Manual alerts can also be generated and sent. All alerts are sent using the Gamma-ray bursts Coordinates Network (GCN) [10] normalized format, allowing easy implementation of connections to additional telescopes. Information about the event that triggered the alert, i.e. a unique identifier, the time and the celestial coordinates, the number of hits used in the reconstruction and the track reconstruction quality are sent to the optical telescopes network at the time of the alert.

\section{The alert criteria}

The criteria for the TAToO trigger are based on the features of the neutrino signal from the expected sources. Several models predict the production of high energy neutrinos greater than $1 \mathrm{TeV}$ from GRBs [11] and from Core Collapse Supernovae [12]. Under certain conditions, multiplet of neutrinos can be expected [13].

Two online neutrino trigger criteria are currently implemented in the TAToO alert system:

- the detection of at least two neutrino induced muons coming from similar directions within a predefined time window;

- the detection of a single high energy neutrino induced muon.

A basic requirement for the coincident observation of a neutrino and an optical counterpart is that the pointing accuracy of the neutrino telescope should be at least comparable to the field of view of the TAROT and ROTSE telescopes.

\subsection{The online track reconstruction algorithm}

To select the events which might trigger an alert, a fast and robust algorithm is used online to reconstruct tracks from the calibrated data. This algorithm uses an idealized detector geometry which does not rely on the dynamical positioning alignment. As a result, the hits of the three OMs of a storey are grouped and their location assigned to the barycenter of the storey. The storey orientations as well as the line-shape deviations from straight lines are not considered in the online reconstruction. A detailed description of this algorithm and its performance is found in Ref. [9]. The principle is to minimize a $\chi^{2}$ which compares the times of selected hits with the expectation from a Cherenkov signal generated by a muon track. The resulting direction of the reconstructed muon track is available within $10 \mathrm{~ms}$ and the obtained reduced $\chi^{2}, \chi_{\text {red }}^{2}=$ $\chi_{\min }^{2} / N_{\text {dof }}$ with $N_{\text {dof }}$ the number of degrees of freedom, is used as a fit quality parameter to remove badly reconstructed tracks.

\subsection{Neutrino selection criteria}

Atmospheric muons, whose abundance at the ANTARES detector [14] is roughly six orders of magnitude larger than the one of muons induced by atmospheric neutrinos, are the main background for the alerts and have to be efficiently suppressed. Among the surviving events, neutrino candidates with an increased probability to be of cosmic origin are selected [15].

\subsubsection{Atmospheric muon background rejection}

Atmospheric muons resulting from the interaction of cosmic rays with nuclei in the atmosphere represent the main component of the background. Atmospheric muons propagate downgoing through the detector and can be suppressed with an elevation cut, selecting only the upgoing events. However, some badly reconstructed atmospheric muons classified as upgoing may remain, and 
quality cuts are applied to reduce this contamination to an acceptable level.

In order to establish the criteria for our neutrino selection, we have analyzed a subsample of data taken by ANTARES after the completion of the 12-line detector, corresponding to a livetime of 70.3 days. During this period, around 350 upgoing neutrino candidates were reconstructed and have been compared to a Monte Carlo (MC) simulation of atmospheric muons and neutrinos using the same livetime. Downgoing atmospheric muons were simulated with Corsika [16], and normalized to match the data. The primary particle flux was composed of several nuclei according to Ref. [17] and the QGSJET hadronic model [18] was used for the shower development. Upgoing neutrinos were simulated according to the parameterization of the atmospheric neutrino flux from Ref. [19]. Only charged current interactions of neutrinos and antineutrinos were considered. The Cherenkov light produced in the vicinity of the ANTARES detector was propagated taking into account light absorption and scattering in sea water [20]. The angular acceptance, quantum efficiency and other characteristics of the optical modules were taken from Ref. [21].

Fig. 1 shows the distribution of the track fit quality $\left(\chi_{\text {red }}^{2}\right)$ of the minimization procedure for all upgoing events reconstructed with at least two lines. A cut on the track fit quality is applied to reduce the number of atmospheric muons reconstructed as upgoing in the final sample. Because the fit quality is correlated to the number of hits used in the fit, the selection cut on the fit quality parameter is set to a different value according to the number of hits used to reconstruct the event: $\chi_{\text {red }}^{2} \leqslant 1.3+\left[0.04 *\left(N_{\text {hit }}-5\right)\right]^{2}$.

Fig. 2 shows the elevation distributions both for data and simulated atmospheric neutrino and muon samples, after the cut on the track fit quality. Atmospheric muons reconstructed as upgoing are efficiently rejected and a neutrino purity better than $90 \%$ is achieved.

Fig. 3 shows the angular resolution of the online algorithm as a function of the neutrino energy for events reconstructed with different number of lines. This resolution is defined as the median of the space angular difference between the incoming neutrino and the reconstructed neutrino-induced muon. For neutrinos with an energy higher than a few tens of $\mathrm{TeV}$, an angular resolution of $0.4^{\circ}$ is achieved, despite of the approximations related to the detector geometry. For example, the inclination of the ANTARES line for a typical sea current of $5 \mathrm{~cm} / \mathrm{s}$ induces a systematic angular deviation of less than $0.2^{\circ}$.

\subsubsection{Multi-neutrino trigger}

The typical signature of the transient emission of high energy neutrinos is a neutrino burst, i.e. a multiplet of neutrino events

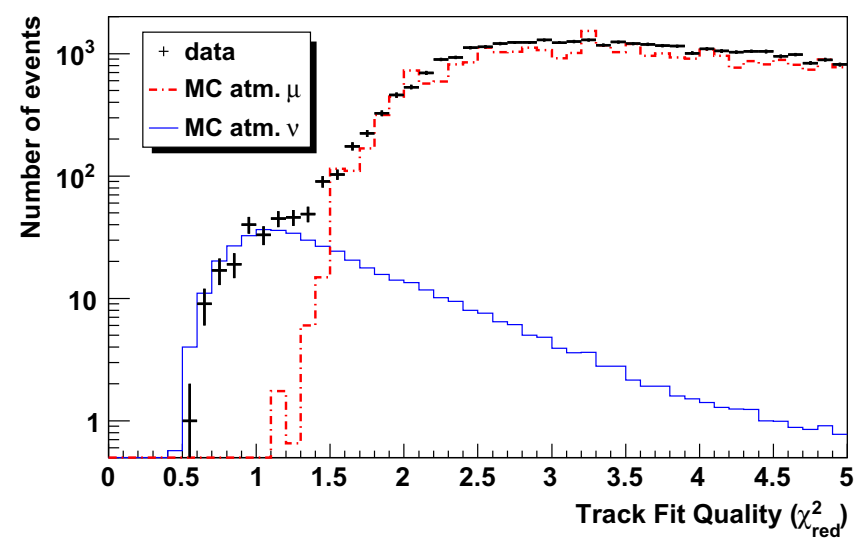

Fig. 1. Track fit quality $\left(\chi_{\text {red }}^{2}\right)$ distribution for all upgoing events reconstructed on at least 2 lines. The atmospheric muon Monte Carlo distribution has been rescaled to match the data.

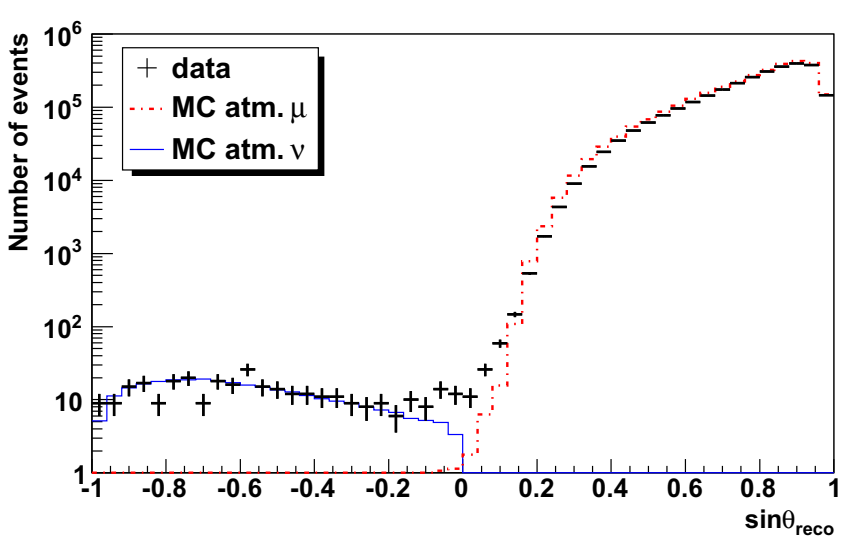

Fig. 2. Elevation angle distribution after a selection cut on the fit quality for all events reconstructed on at least 2 lines.

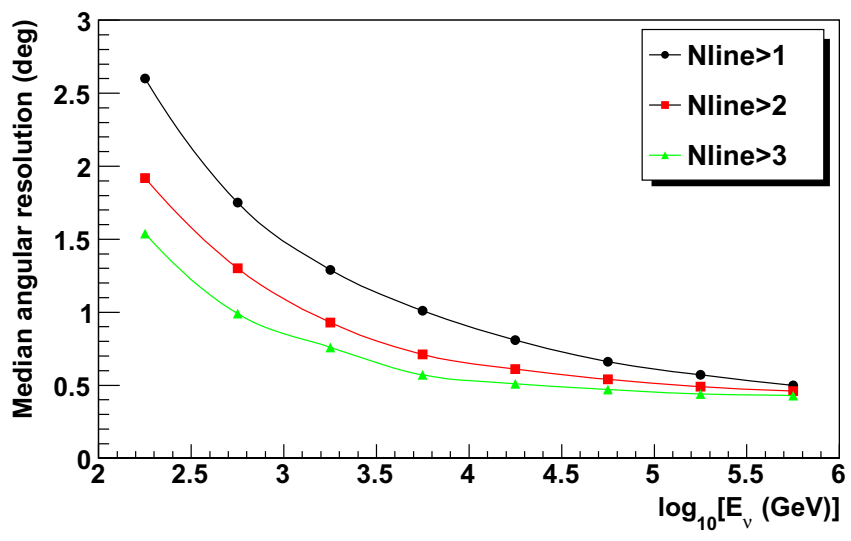

Fig. 3. Angular resolution as a function of the neutrino energy for events with tracks reconstructed with different number of lines.

originating from the source in a short time window. For the multi-neutrino trigger, the minimal cuts providing a pure neutrino sample are used to identify the single neutrinos which belong to the multiplet. This set of cuts is used as a reference for the evaluation of the efficiency for other triggers.

The time window was optimized to include most predictions of the neutrino emission by various models for transient sources. The $3^{\circ}$ angular window was selected to match the convolution of the track reconstruction angular resolution and the field of view of the robotic optical telescopes $\left(\approx 2^{\circ} \times 2^{\circ}\right)$. For the multi-neutrino trigger, the angular resolution is equal to the resolution of each single neutrino, divided by the square root of the multiplicity.

The accidental coincidence rate due to background events, from two uncorrelated upgoing atmospheric neutrinos, is estimated to be $7 \times 10^{-3}$ coincidences per year with the full ANTARES detector. With such a small background, the detection of a doublet (triplet) in ANTARES would have a significance of about 3 (5) sigma.

\subsubsection{High energy event trigger}

Since the neutrino energy spectrum for signal events is expected to be harder than for atmospheric neutrinos, a cut on the reconstructed energy efficiently reduces the atmospheric neutrino background while most of the signal events are kept.

The selection of the alert candidates is based on two simple energy estimators: the number of storeys used in the track fit and the total amplitude (in photoelectrons) of the hits in the storeys. Figs. 4 


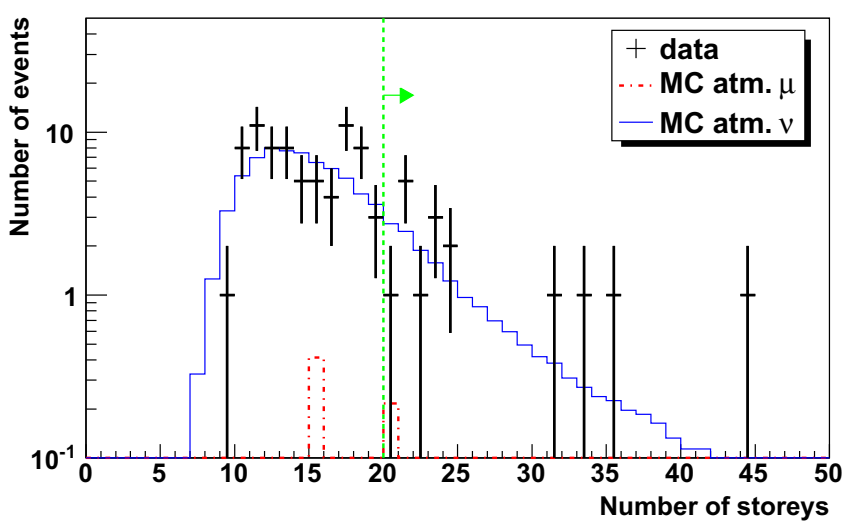

Fig. 4. Distribution of number of storeys having at least one hit used in the reconstruction, for data and Monte Carlo upgoing events reconstructed on at least three lines. The vertical line indicates the alert selection criterium.

and 5 show the distributions of the number of storeys and the amplitude, respectively, both for data and Monte Carlo samples.

The event selection for the high energy trigger has been tuned on atmospheric neutrinos in order to obtain a false alarm rate of about 25 alerts per year. This rate was agreed between ANTARES and the optical telescope collaborations. A requirement of at least 20 storeys on at least three lines and an amplitude greater than 180 photoelectrons will select around 25 high energy events per year with the full 12 line configuration of the ANTARES detector. These cuts allow to select around $86 \%$ of typical GRB neutrinos [11] and around 5\% of typical core-collapse SNe neutrinos [12] while more than $98 \%$ of the atmospheric neutrino and muon background is rejected.

\subsubsection{Trigger performance}

The performance of these two triggers has been studied using a neutrino Monte Carlo generated with an $E^{-2}$ energy spectrum. The TAToO alert criteria efficiently select neutrinos of energies above $1 \mathrm{TeV}$ for the multi-neutrino trigger, and above $10 \mathrm{TeV}$ for the single high energy trigger. Fig. 6 shows the ANTARES effective area for a neutrino passing both alert criteria.

The pointing accuracy of the neutrino telescope has two contributions: the angle between the incoming neutrino and the resulting muon, and the angle between the muon and the reconstructed trajectory. The former contribution is due to kinematics: the more energetic the neutrino, the more co-linear the resulting muon will tend to be with the neutrino direction. The latter contribution is

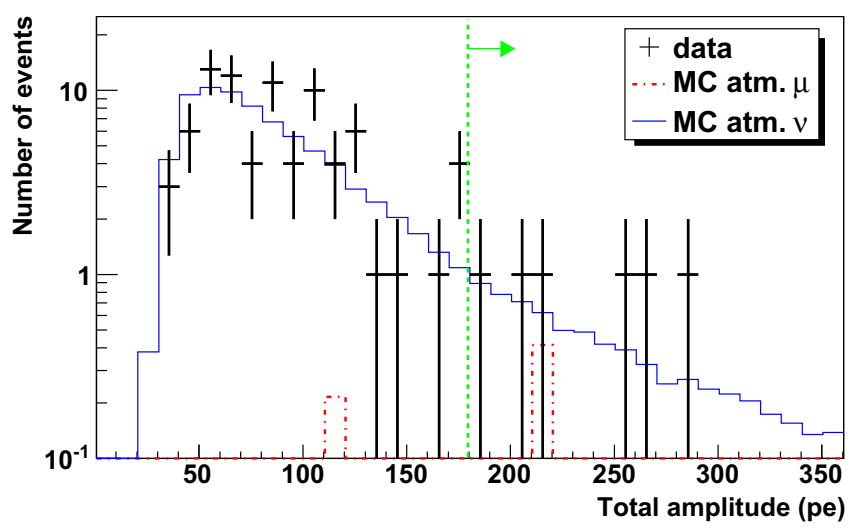

Fig. 5. Total amplitude distribution in photoelectrons (pe) for data and Monte Carlo upgoing events reconstructed on at least three lines. The vertical line indicates the alert selection criterium.

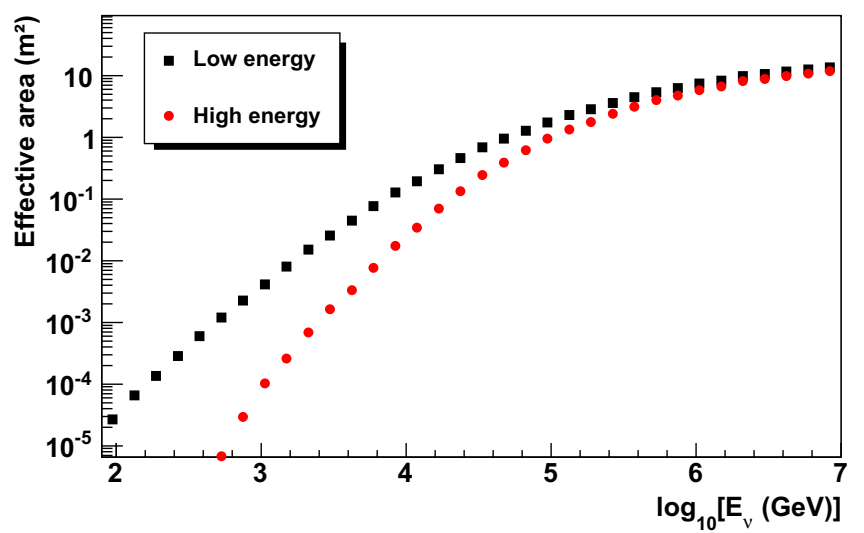

Fig. 6. Neutrino effective area as a function of the true neutrino energy for the events selected by the two alert triggers.

determined by the performance of the track reconstruction algorithm. The bidimensional point spread function expected for neutrino events selected by the single high energy trigger is shown in Fig. 7. As an illustration, $\sim 80 \%$ of the events are reconstructed within the field of view (FOV) of the TAROT or ROTSE telescopes. Fig. 8 shows the dependence of this fraction on the neutrino energy.

\section{Determination of the refined direction}

Although the pointing accuracy of the online reconstruction algorithm is suitable for the field of view of the telescopes used for the follow-up, the use of the detailed knowledge of the detector geometry can further improve the determination of the neutrino direction. For this purpose, we use the standard ANTARES offline reconstruction algorithm [22].

Since the ANTARES lines are not rigid structures, sea currents can move the top buoy by several meters, and distort the line positions from a vertical line geometry, thus affecting the direction of the reconstructed muon trajectory.

In order to achieve the best track reconstruction performance, it is necessary to monitor the relative positions of all OMs with an

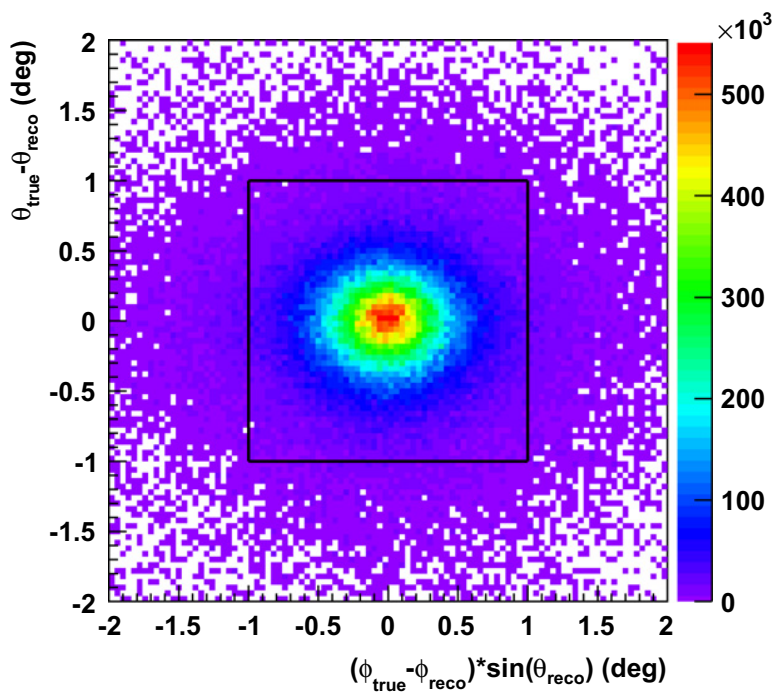

Fig. 7. Bidimensional point spread function. The black solid-line square corresponds to the TAROT field of view $\left(\approx 2^{\circ} \times 2^{\circ}\right)$. 


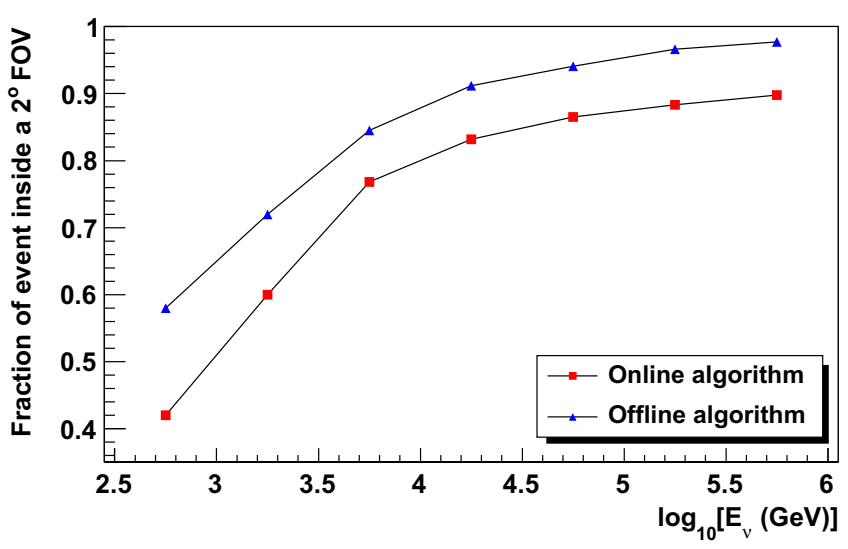

Fig. 8. Fraction of events inside a field of view of $2^{\circ} \times 2^{\circ}$ as a function of the energy of the event, assuming that the tracks originated from the center of the field of view, for both online and offline reconstruction algorithms.

accuracy of better than $20 \mathrm{~cm}$, equivalent to $1 \mathrm{~ns}$ timing precision. In addition, a precise absolute orientation of the whole detector is necessary to point to individual neutrino sources in the sky. Two independent monitoring systems are used to attain the required accuracy:

- A high frequency long baseline acoustic system giving the 3D position of hydrophones placed along the line. These positions are obtained by triangulation from emitters anchored at the bases of the lines.

- A set of tiltmeter-compass sensors giving the local tilt angles of each OM storey with respect to the horizontal plane (pitch and roll) as well as its orientation with respect to the Earth's magnetic field (heading).

In order to obtain a quasi-online precise detector geometry (within a delay of typically few tens of minutes), the shape of the detector lines is derived from a model which estimates the mechanical behavior under the influence of the sea water flow, obtained from online measurements of the sea current. The positions of the OMs are then calculated by combining the line shape with the measurements of the tilt and orientation angles of the storeys given by the tiltmeter-compass sensors.

\subsection{The offline likelihood fit}

The offline reconstruction algorithm derives the muon track parameters that maximize a likelihood function built from the difference between the expected and the measured arrival time of the hits from the Cherenkov photons emitted along the muon track. This maximization [22] takes into account the Cherenkov photons that scatter in the water and the additional photons that are generated by secondary particles (e.g. electromagnetic showers created along the muon trajectory).

The value of the log-likelihood per degree of freedom $(\Lambda)$ from the track reconstruction fit is a measure of the track fit quality and is used to reject badly reconstructed events, such as atmospheric muons that are mis-reconstructed as upgoing tracks. The distribution of the variable $\Lambda$ is shown in Fig. 9 for both data and Monte Carlo events. Only tracks reconstructed with $\Lambda>-5.2$ are kept for the determination of the refined track direction.

\subsection{Offline reconstruction performance}

Fig. 10 shows the median Monte Carlo computed angular resolution as a function of the neutrino energy for events selected by the high energy alert trigger, for both online and offline algorithms. The improvement obtained with the offline algorithm is clearly visible at low energies. The angular resolution for neutrino energies above $10 \mathrm{TeV}$ is $0.5^{\circ}$ for the online algorithm and $0.35^{\circ}$ for the offline algorithm. As illustrated in Fig. 7, $92 \%$ of the signal events are reconstructed within a FOV of $2^{\circ} \times 2^{\circ}$.

\section{Optical follow-up procedure}

The optical follow-up strategy is based on short-term observations (for rapidly fading sources) and a long-term follow-up (mainly for core collapse SNe searches). Once the alert is sent at date $T_{0}$, optical images are collected as soon as possible by the available telescopes. The fast slewing of the small robotic telescopes allow the collection of images from 5 to $10 \mathrm{~s}$ after the reception of the alert. The follow-up procedure, which extends over 1 month with images taken each night during the first week following the date $T_{0}$ of the alert, makes use of the refined direction. For these observations, an additional constraint is added: if the refined direction is more than $0.5^{\circ}$ away from the initial direction and has a poor offline reconstruction quality, the follow-up of the alert is canceled. Otherwise, the pointing direction of the telescopes is updated with the refined coordinates and all subsequent images are centered around that direction.

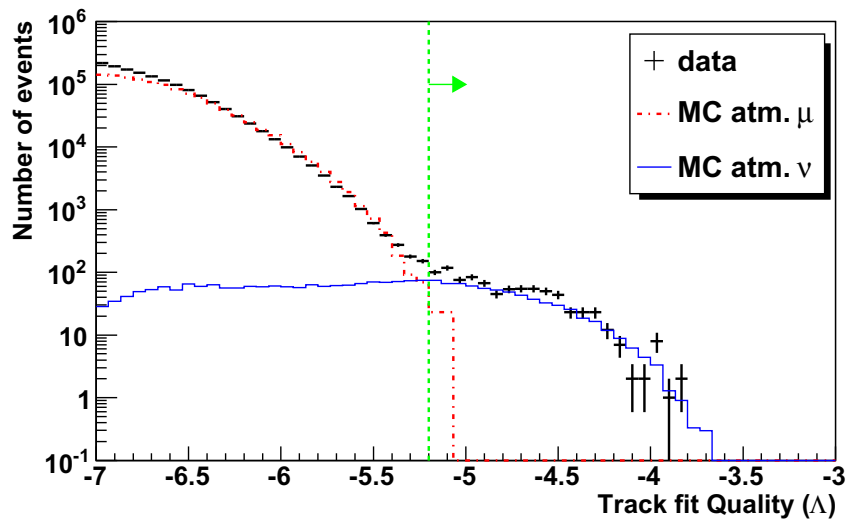

Fig. 9. Track fit quality $(\Lambda)$ distribution for upgoing events in both data and atmospheric Monte Carlo samples.

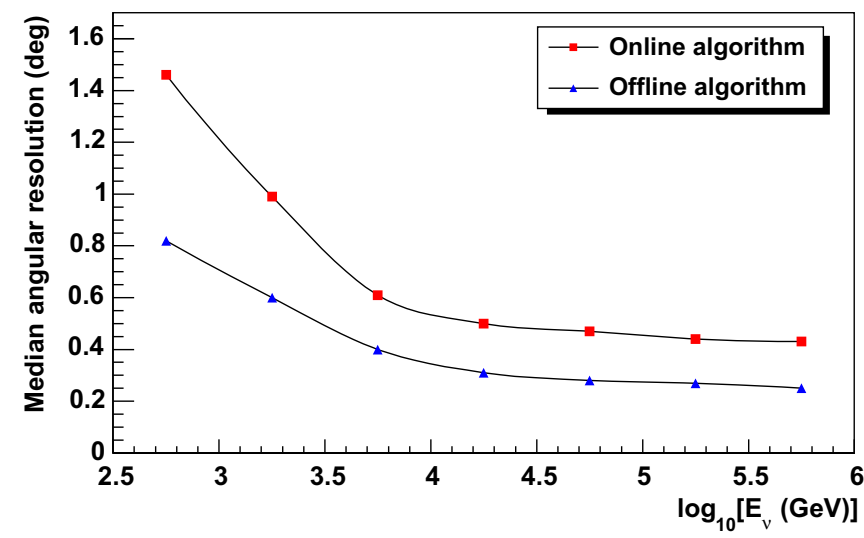

Fig. 10. Angular resolution obtained for both online and offline reconstructions as a function of the neutrino energy. 


\section{Summary}

The method used by the ANTARES collaboration to implement the search for coincidence between high energy neutrinos and transient sources followed by small robotic telescopes has been presented. Of particular importance for this alert system are the ability to reconstruct online the neutrino direction and to reject efficiently the background. With the described ANTARES alert sending capability, the connected optical telescopes can start taking images with a latency of the order of one minute, which will be reduced to about $15 \mathrm{~s}$ in the near future. The precision of the direction of the alert is much better than one degree. The quasionline availability of a refined direction obtained using the measured geometry of the ANTARES detector further improves the quality and efficiency of the alert system.

The alert system is operational since February 2009, and as of December 2010, 27 alerts have been sent, all of them triggered by the high energy selection criterium. No doublet trigger has been recorded yet. After a commissioning phase in 2009, all alerts had an optical follow-up in 2010, and the live time of the system over this year is strictly equal to the one of the ANTARES telescope, namely $87 \%$. These numbers are consistent with the expected trigger rate, after accounting for the duty cycle of the neutrino telescope.

The optical follow-up of neutrino events significantly improves the perspective for the detection of transient sources. A confirmation by an optical telescope of a neutrino alert will not only provide information on the nature of the source but also improve the precision of the source direction determination in order to trigger other observatories (for example telescopes for spectroscopic redshift measurements). The program for the follow-up of ANTARES neutrino events is already operational with the TAROT and ROTSE telescopes and results based on analysis of the optical images will be presented in a forthcoming paper. This technique could be extended to observations in other wavelength regimes such as Xray or radio.

\section{Acknowledgments}

The authors acknowledge the financial support of the funding agencies: Centre National de la Recherche Scientifique (CNRS), Commissariat à l'énergie atomique et aux énergies alternatives (CEA), Agence National de la Recherche (ANR), Commission Européenne (FEDER fund and Marie Curie Program), Région Alsace (contrat CPER), Région Provence-Alpes-Côte d'Azur, Département du Var and Ville de La Seyne-sur-Mer, France; Bundesministerium für Bildung und Forschung (BMBF), Germany; Istituto Nazionale di Fisica Nucleare (INFN), Italy; Stichting voor Fundamenteel Onderzoek der Materie (FOM), Nederlandse organisatie voor Wetenschappelijk Onderzoek (NWO), the Netherlands; Council of the President of the Russian Federation for young scientists and leading scientific schools supporting grants, Russia; National Authority for Scientific Research (ANCS), Romania; Ministerio de Ciencia e Innovación (MICINN), Prometeo of Generalitat Valenciana and MultiDark, Spain. We also acknowledge the technical support of Ifremer, AIM and Foselev Marine for the sea operation and the CC-IN2P3 for the computing facilities.

This work has been financially supported by the GdR PCHE in France. We want to thank M. Kowalski for discussions on the neutrino triggers and the organization of the optical follow-up.

\section{References}

[1] T. Piran, Phys. Rep. 314 (1999) 575.

[2] M. Kowalski, A. Mohr, Astropart. Phys. 27 (2007) 533.

[3] E. Aslanides et al., ANTARES Collaboration, <astro-ph/9907432>; P. Coyle et al., in: Proc. 31st ICRC, Lódź 2009. <arXiv:1002.0701v1>; M. Ageron et al., <arXiv:1104.1607v1[astro-ph.IM]>.

[4] D. Dornic et al., Nucl. Instrum. Methods A 626-627 (2011) S183; S. Basa et al., Nucl. Instrum. Methods A 602 (2009) 275.

[5] M. Boer et al., Astron. Astrophys. Suppl. Ser. 138 (1999) 579.

[6] C.W. Akerlof et al., Public. Astron. Soc. Pac. 115 (2003) 132.

[7] J.A. Aguilar et al., ANTARES Collaboration, Nucl. Instrum. Methods A 570 (2007) 107.

[8] J.A. Aguilar et al., ANTARES Collaboration, Astropart. Phys. 34 (2011) 539.

[9] J.A. Aguilar et al., ANTARES Collaboration, Astropart. Phys. 34 (2011) 652.

[10] GCN network. <http://www.gcn.gsfc.nasa.gov>.

[11] E. Waxman, J. Bahcall, Phys. Rev. Lett. 78 (1997) 2292;

P. Meszaros, E. Waxman, Phys. Rev. Lett. 87 (2001) 171102;

C. Dermer, A. Atoyan, Phys. Rev. Lett. 91 (2003) 071102;

S. Razzaque, P. Meszaros, E. Waxman, Phys. Rev. Lett. 90 (2003) 241103.

[12] S. Ando, J. Beacom, Phys. Rev. Lett. 95 (2005) 061103.

[13] S. Razzaque, P. Meszaros, E. Waxman, Phys. Rev. Lett. 94 (2005) 109903.

[14] J.A. Aguilar et al., ANTARES Collaboration, Astropart. Phys. 34 (2010) 179

[15] J.A. Aguilar et al., ANTARES Collaboration, Phys. Lett. B696 (2011) 16.

[16] D. Heck et al., Report FZKA 6019, Forschungszentrum Karlsruhe, 1998.

[17] J.R. Hörandel, Astropart. Phys. 19 (2003) 193.

[18] N.N. Kalmykov, S.S. Ostapchenko, Yad. Fiz. 56 (1993) 105; N.N. Kalmykov, S.S. Ostapchenko, Phys. At. Nucl. 56 (3) (1993) 346.

[19] G. Barr et al., Phys. Rev. D39 (1989) 3532; V. Agrawal et al., Phys. Rev. D53 (1996) 1314.

[20] J.A. Aguilar et al., ANTARES Collaboration, Astropart. Phys. 23 (2005) 131.

[21] P. Amram et al., Nucl. Instrum. Methods A 484 (2002) 369.

[22] A. Heijboer, Ph.D. thesis, Universiteit van Amsterdam, Amsterdam, The Netherlands. <http://www.antares.in2p3.fr/Publications/thesis/2004/AartHeijboer-phd.pdf $>$. 